\title{
The irritable hip: immediate ultrasound guided aspiration and prevention of hospital admission
}

\author{
A Michelle Fink, Laurence Berman, Dennis Edwards, S Kim Jacobson
}

\begin{abstract}
A protocol for the management of irritable hip was assessed; this protocol avoids hospital admission while detecting all other serious causes of hip pain, in particular septic arthritis, at the earliest possible opportunity.

Fifty children with painful hips were studied prospectively with immediate ultrasound guided aspiration and Gram stain of all hip effusions. Bone scintigraphy performed at an early stage was reserved for patients with unremitting symptoms.

Thirty six hips were aspirated. Only two patients were admitted. The final diagnoses were transient synovitis (45 cases), Perthes' disease (three cases), fracture (one case), and septic arthritis (one case). The single case of hip sepsis was diagnosed on presentation.

The traditional approach to management is questioned and the advantages of the protocol highlighted, including earliest diagnosis of sepsis and other serious pathology, avoidance of hospital admission, and the relief of pain by joint decompression.
\end{abstract}

(Arch Dis Child 1995; 72: 110-114)

Keywords: irritable hip, ultrasound, aspiration.

The painful hip is one of the most common causes of acute paediatric orthopaedic hospital admission in Great Britain. Although most are retrospectively labelled as having had transient synovitis, a diagnosis of exclusion, ${ }^{1}$ patients are frequently admitted for observation because of concern that there may be a more serious underlying diagnosis, in particular a septic hip. A recent paper emphasised that many of these patients may be managed as outpatients and discussed symptoms and signs that may distinguish the patients at risk from hip sepsis, possibly reducing hospital admissions by $85 \% .^{2}$ Others have noted that the symptoms and signs of hip sepsis and a sterile irritable hip may be identical, ${ }^{3}$ but sepsis requires immediate treatment. ${ }^{4-6}$ Despite hospital admission, delayed diagnosis of septic arthritis occurs, as hip aspiration and culture is often only undertaken when patients have worsening or persistent systemic symptoms or abnormal laboratory findings. Clinical and laboratory parameters such as irritability, fever, peripheral leucocyte count, and erythrocyte sedimentation rate (ESR) do not reliably predict hip sepsis. ${ }^{67}$ Infection can only be excluded by the culture of synovial fluid that can be accurately identified and aspirated using ultrasound guidance. $^{8}$

The authors evaluated a strategy for the earliest possible exclusion of joint sepsis in all children presenting with a painful hip. This protocol also obviates the need for hospital admission, yet detects other important pathology that may present with acute hip pain.

\section{Patients and methods}

Fifty consecutive children presenting with acute hip pain were prospectively studied. The 33 boys and 17 girls were referred by their general practitioner or presented directly to the casualty department. Ages ranged from 1 to 10 years (mean $=5.4$ years). After examination, Emla topical anaesthetic cream (Astra) was applied to the anterior skin crease of the painful hip, blood was taken for leucocyte count and ESR, and pelvic radiography which included a frog lateral view was performed.

The patient then underwent immediate sonography of both hips, using a $7.5 \mathrm{MHz}$ linear array transducer (Aloka 650 or Toshiba 270), to identify any hip effusion. Longitudinal images of the joint capsule and psoas bursa anterior to the femoral head and neck were obtained. If a joint effusion was present, it was immediately aspirated after careful explanation of the procedure to parents and child. Aspirations were performed under strict aseptic conditions using a technique developed by the authors (L Berman et al; Br $\mathcal{F}$ Radiol 1995: in press). This involves careful marking of surface landmarks but no scanning during the aspiration itself, as sterile conditions are then more difficult to maintain. Sedation was not required. The aspirate was sent for immediate microscopy and Gram stain. Specimens were inoculated into a brain-heart infusion broth and onto blood and chocolate agar which were incubated at $37^{\circ} \mathrm{C}$ in $5 \%$ carbon dioxide for a total of five days.

If the immediate Gram stain did not reveal organisms the child was discharged provided 
there were suitable domestic arrangements and no major systemic symptoms. Contact with the general practitioner was established in all cases and provision made for urgent recall should the cultures subsequently prove positive. An outpatient appointment within one week was arranged.

At the outpatient review all patients whose symptoms had not improved underwent repeat sonography with aspiration of fluid if present, and a technetium-99m methylene diphosphonate bone scan was requested. All patients were followed up until they had made a full recovery.

\section{Results}

INITIAL SCANS AND ASPIRATIONS

Of the 50 children, $36(72 \%)$ had detectable effusions that were aspirated. Twenty eight of the 36 reported immediate relief of hip pain after aspiration.

\section{INITIAL MICROSCOPY}

Immediate Gram stain demonstrated Gram positive cocci in one case.

In 35 cases (97\% of all effusions) no organisms were seen on microscopy, although in several cases the aspirate appeared macroscopically purulent and polymorphs were seen on microscopy in 31 of the 35 (89\%).

\section{CULTURES}

In the single patient with a positive Gram stain, Streptococcus pyogenes was subsequently cultured. All 35 in whom microscopy did not reveal any organisms remained sterile after five days, and therefore no patient with an initially normal Gram stain was recalled for antibiotic treatment.

BLOOD TESTS

The leucocyte count ranged from $5 \cdot 1$ to $22.5 \times 10^{9} / 1 \quad($ mean $=10 \cdot 2)$, and was greater than $15 \times 10^{9} / 1$ in seven cases. In the only case of hip sepsis the leucocyte count was $20.4 \times 10^{9} / 1$. The ESR ranged from 4-76 $\mathrm{mm} /$ hour $($ mean $=15)$ and was greater than 20 $\mathrm{mm} /$ hour in 17 cases. In the single case of sepsis the ESR was $30 \mathrm{~mm} /$ hour.

\section{IMAGING}

Plain radiographs

All radiographs were assessed by a single experienced radiologist and were evaluated for bone and joint abnormalities other than hip effusions for which ultrasound was used. The radiograph was considered normal in 46 cases. There were three abnormal radiographs. Two patients had features of established Perthes' disease and one had a suspicion of undisplaced impacted femoral fracture. In one case a slightly irregular femoral capital epiphysis suggested early Perthes' disease but a bone scan and subsequent clinical course were normal.

\section{Bone scintigraphy}

Four isotope bone scans were performed of which two were abnormal. One abnormal scan resulted from a patient with a normal radiograph where hip pain had not resolved by the outpatient attendance. The scan demonstrated the 'cold hip' sign of early Perthes' disease, ${ }^{9}$ and the patient subsequently developed characteristic radiological changes. The second abnormal bone scan confirmed the diagnosis in the child with the suspected femoral fracture.

Of the two normal scans, one was undertaken in the child with equivocal signs of Perthes' disease on the plain radiograph. The remaining normal scan was in a patient admitted for severe systemic symptoms despite the aspiration of a sterile effusion. The scan was performed to exclude osteomyelitis.

\section{HOSPITAL ADMISSIONS}

Two patients (4\%) in this study were admitted to hospital. One was the child with the infected hip (leucocyte count $20.4 \times 10^{9} / 1$, ESR 30 $\mathrm{mm}$ /hour); she subsequently grew $S$ pyogenes on blood culture. The second admission was on the basis of severe systemic symptoms and fever. The leucocyte count was $7 \cdot 8 \times 10^{9} / 1$ and ESR $76 \mathrm{~mm} /$ hour. The radiograph was normal and no effusion was demonstrated on presentation, but fluid was detected and aspirated 24 hours later. The Gram stain and culture of the aspirate were negative and isotope bone scan was normal. Blood cultures were sterile. No antibiotic treatment was given, he was discharged after five days and had made a full recovery within two weeks.

FINAL DIAGNOSES $(\mathrm{N}=50)$

The final diagnoses were transient synovitis (45 cases), Perthes' disease (three cases), femoral fracture (one case), and septic arthritis (one case).

\section{Discussion}

Ultrasound demonstrates and facilitates the aspiration of hip effusions but has not been integrated into a more logical approach to patient management. A policy of aspirating all effusions may appear needlessly invasive, particularly as most children presenting with hip pain have transient synovitis which is a benign, self limiting condition, the treatment of which is symptomatic. ${ }^{1}$ It is usually managed with admission, observation, traction, and analgesia. In agreement with a recent study, ${ }^{2}$ the authors would question all aspects of this traditional management that dates from a presonographic era when open arthrotomy under general anaesthesia was required to obtain synovial fluid. This radical procedure could still result in a dry tap.

Hospital admission for observation is still the rule with a mean duration of 4-7 days. ${ }^{1} 1011$ Presumably the main reason for admission is clinical concern that there may be a more serious underlying condition than transient synovitis, in particular septic arthritis. ${ }^{12}$ If 
sepsis were excluded admission would be unnecessary.

In their recent review of admissions at Southampton, Taylor and Clarke suggested that admission may be reserved for patients with fever $\geqslant 38^{\circ} \mathrm{C}$, ESR $\geqslant 20 \mathrm{~mm} /$ hour, and severe pain or spasm. ${ }^{2}$ This approach is not supported by other authors who noted that simple parameters such as temperature, leucocyte count, and ESR are of little use in determining which patients have joint sepsis. ${ }^{67}$ In a series of 45 children with a proven septic hip, one third were afebrile and a third had a normal leucocyte count. ${ }^{6}$

Hip sepsis is an orthopaedic emergency that can result in rapid permanent joint destruction. The single most important prognostic factor is the speed with which the diagnosis is made and antibiotic treatment instituted, a delay of more than four days after the onset of symptoms being associated with poor outcome. ${ }^{4-6}$ The only method of confirming or refuting the diagnosis of septic arthritis is the culture of synovial fluid. Microscopy and Gram staining of the aspirate provides a rapid method for the detection of organisms and may be useful when the patient has already received antibiotics or before the results of culture are available. ${ }^{5}$

High resolution ultrasonography can accurately detect the presence of even very small joint effusions, 81314 but it does not differentiate between sterile and infected effusions. $^{8} 1315-18$ Obtaining synovial fluid is a simple, relatively non-invasive procedure, the aspiration being performed in the ultrasound department under topical local anaesthesia without the need for sedation. In the present study there were no unsuccessful aspirations.

Analgesia may be considered an indication for admission. Pain relief is traditionally achieved using skin traction, but this has been shown in a recent study not to influence outcome. ${ }^{1}$ The present study confirms previous reports that the need for analgesia is reduced by decompressing the joint. 121819 Most patients in the present study reported immediate relief of pain after aspiration.

There are other important causes of acute hip pain that may have a similar presentation to transient synovitis which include Perthes' disease, slipped femoral capital epiphysis, osteomyelitis, and bone tumours. ${ }^{7} 1020$ Radiographs may at times be normal in these conditions. Using the protocol suggested in the present study, patients undergo a bone scan within a week if their symptoms persist. It is unlikely that these diagnoses will be delayed as scintigraphy will detect early Perthes' disease, ${ }^{1121}$ fractures, osteomyelitis, ${ }^{10}$ and tumours not visible on radiographs. There is debate about the need for plain radiographs in children with acute hip pain. ${ }^{22}$ The examinations are often normal and are far less sensitive for the detection of hip effusions than ultrasound. ${ }^{23}$ The present study did not address this issue, although the radiograph provided the diagnosis in two of the three cases of Perthes' disease as well as in the child with the femoral fracture. Arguably all would have been detected on the bone scan performed for continued symptoms had no radiograph been obtained. Another approach could be to obtain radiographs in patients with delayed symptoms, before proceeding to bone scan. There was no case of slipped femoral capital epiphysis in the present series, but there have been several reports of the use of ultrasound to detect this condition as well as Perthes' disease. ${ }^{24-28}$ At present ultrasound is not used to make these diagnoses in the authors' hospital.

In a recent paper several patients are reported to have undergone open surgical arthrotomy on the basis of their symptoms and turbid ultrasound guided hip aspirates alone. ${ }^{29}$ The need for arthrotomy whether or not the aspirate is turbid or even infected can be questioned. In the present study many children had a raised leucocyte count and ESR, and most aspirates contained pus cells, some appearing macroscopically purulent; yet in only one was this associated with infection of the joint which was diagnosed immediately by Gram stain and confirmed by culture. Treatment was successful without surgery. Regarding the concern that hip aspiration may introduce infection into a previously sterile joint, there is now a large number of reported cases of ultrasound guided hip aspiration using a variety of techniques and presumably many more have been conducted in normal practice that have not been presented in the literature. The authors are not aware of any reported account of hip infection attributed to this procedure.

Taylor and Clarke in their recent review, describe two cases of Perthes' disease that only became apparent one month and six months after admission with an irritable hip. ${ }^{2}$ They also describe three interesting patients with persistent pain and a cold hip sign on bone scan who never developed Perthes' disease, suggesting that there is forme fruste that presents as an irritable hip but may resolve spontaneously. There is speculation whether transient synovitis is the latent phase of Perthes' disease, the sterile effusion causing tamponade of the blood supply to the femoral head. The few protagonists of this theory advocate immediate aspiration of all hip effusions to reduce the intra-articular pressure. ${ }^{3031}$ Although it was not the authors' primary purpose, the regimen suggested would achieve this.

In conclusion, immediate ultrasound with aspiration of all hip effusions obviated the need for admission in 48 out of 50 patients. One case of septic arthritis was diagnosed at the earliest opportunity. In addition no child underwent general anaesthesia or surgery. Management of the painful hip should include immediate ultrasound with the aspiration of all effusions. This will eliminate delay in the diagnosis of septic arthritis, avoid hospital admission in most children, and frequently provide immediate relief of pain. The suggested protocol provides a 'safety net' of bone scintigraphy within a week of presentation for those patients with persistent symptoms. This will identify other important causes of hip pain. 
1 Briggs RD, Baird KS, Gibson PH. Transient synovitis of the hip joint. F $R$ Coll Surg Edinb 1990; 35: 48-50.

2 Taylor GR, Clarke NMP. Management of irritable hip: a review of hospital policy. Arch Dis Child 1994; 71: 59-63.

3 Paterson D. Septic arthritis of the hip joint. Orthop Clin North Am 1978; 9: 135-42.

4 Morrey BF, Bianco AJ, Rhodes KH. Suppurative arthritis of the hip in children. F Bone foint Surg Am 1976; 58: 388-92.

5 Jackson MA, Nelson JD. Etiology and medical management of acute supurative bone and joint infections in pediatric patients. $\mathcal{F}$ Pediatr Orthop 1982; 2: 313-23.

6 Bennett OM, Namnyak SS. Acute septic arthritis of the hip joint in infancy and childhood. Clin Orthop 1992; 281: joint in

7 Del Beccaro MA, Champoux AN, Bockers T, Mendelman PM. Septic arthritis versus transient synovitis of the hip: the value of screening laboratory tests. Ann Emerg Med 1992; 21 : 1418-22.

8 Marchal GJ, Van Holsbeeck MT, Raes M, et al. Transient synovitis of the hip in children: role of US. Radiology 1987; 162: 825-8.

9 Uren RF, Howman-Giles R. The 'cold hip' sign on bone scan. A retrospective review. Clin Nucl Med 1991; 16: 553-6.

10 Choban S, Killian JT. Evaluation of acute gait abnormalities in preschool children. $\mathcal{F}$ Pediatr Orthop 1990; 10: 74-8.

11 Gopakumar TS, Vaishya R, Klenerman L, Carty H. The role of ultrasound and isotope scanning in the managerole of ultrasound and isotope scanning in the manage
ment of irritable hips. Eur $\mathcal{F}$ Radiol 1992; 15: 113-7.

12 Hill SA, MacLarnon JC, Nag D. Ultrasound-guided aspiration for transient synovitis of the hip. $\mathcal{F}$ Bone foint Surg $B r$ 1990; 72: 852-3.

13 Miralles M, Gonzalez G, Pulpeiro JR, et al. Sonography of the painful hip in children: 500 consecutive cases. AfR 1989; 152: 579-82.

14 Bialik V, Volpin G, Jerushalmi J, Stein H. Sonography in the diagnosis of painful hips. Int Orthop 1991; 15: 155-9.

15 Wilson DJ, Green DJ, MacLarnon JC. Arthrosongraphy of the painful hip. Clin Radiol 1984; 35: 17-9.

16 Alexander JE, Seibert JJ, Glasier CM, et al. High-resolution hip ultrasound in the limping child. Fournal of Clinical Ultrasound 1989; 17: 19-24.

17 Egund N, Wingstrand $\mathrm{H}$. Pitfalls in ultrasonography of hip joint synovitis in the child. Acta Radiol 1989; 30: 375-9.

18 Shiv VK, Jain AK, Taneja K, Bhargava SK. Sonography of hip joint in infective arthritis. Fournal of the Canadian hip joint in infective arthritis. Fournal

19 Rauch G, Schuler P, Wirth T, Griss P, Dorner P. Diagnosis and therapy of coxitis fugax with special reference to the value of ultrasonography assisted diagnosis and hip joint puncture. Zeitschrift für Orthopädie und ihre Grenzgebiete 1993; 131: 105-10.

20 Weinberg JR, Berman L, Dootson G, Mitchell R. Pubic osteomyelitis presenting as irritable hip. Postgrad Med $\mathcal{J}$ 1987; 63: 301-2.

21 Royle SG, Galasko CSB. The irritable hip. Scintigraphy in 192 children. Acta Orthop Scand 1992; 63: 25-8.

22 Meradji M, Diepstraten AFM. Coxitis fugax: sonographisches und radiologisches Bild in 65 Fällen. Radiologe 1988; 28: $473-8$.

23 Bickerstaff DR, Neal LM, Booth AJ, Brennan PO, Bell MJ. Ultrasound examination of the irritable hip. $\mathcal{F}$ Bone foint Surg Br 1990; 72: 549-53.

24 Dörr U, Zieger M, Hauke H. Ultrasonography of the painful hip. Prospective studies in 204 patients. Pediatr Radiol 1988; 19: 36-40.

25 Terjesen T. Ultrasonography for diagnosis of slipped capital femoral epiphysis: comparison with radiography in 9 cases. Acta Orthop Scand 1992; 63: 653-7.

26 Castriota-Scanderbeg A, Orsi E. Slipped capital femoral epiphysis: ultrasonographic findings. Skeletal Radiol 1993; 22: 191-3.

27 Futami T, Kasahara Y, Suzuki S, Ushikubo S, Tsuchiya T. Ultrasonography in transient synovitis and early Perthes' disease. F Bone foint Surg Br 1991; 73: 635-9.

28 Terjesen $\mathrm{T}$. Ultrasonography in the primary evaluation of patients with Perthes' disease. F Pediatr Orthop 1993; 13: 437-43.

29 Zawin JK, Hoffer FA, Rand FF, Littlewood Teele R. Joint effusion in children with an irritable hip: US diagnosis and aspiration. Radiology 1993; 187: 459-63.

$30 \mathrm{Kemp} \mathrm{HB}$. Perthes' disease: the influence intracapsular tamponade on the circulation in the hip joint of the dog. tamponade on the circulation in
Clin Orthop 1981; 156: 105-14.

31 Hasegawa $\mathrm{Y}$, Ito $\mathrm{H}$. Intracapsular pressure in hip synovitis in children. Acta Orthop Scand 1991; 62: 333-6.

\section{Commentary}

The principal claim of Fink et al, stated in the title to their paper, is reasonable in itself, but may well prove inadequate to meet the wider variety of pathology which presents itself in larger series of irritable hip patients. The authors presuppose that 'all other serious causes of hip pain, in particular septic arthritis', will be detected by ultrasound by the finding of a hip effusion which may be diagnostically aspirated. In the first instance the 'accuracy' of sonography in the detection of hip effusions was questioned by McGoldrick et al, in the only single blind trial of this technique reported in the literature. ${ }^{1}$ Forty six children underwent aspiration; two were found to have an effusion that had not been detected by ultrasound. Even accepting its sensitivity, there is still the premise that 'other serious causes of hip pain' presenting as irritable hip necessarily coexist with an effusion. In our series of 509 consecutive children with painful hips, ${ }^{2} 21$ provided to have a local septic condition, including nine with osteomyelitis affecting the adjacent pelvis, none of whom are likely to have had an effusion. Furthermore, it is unknown whether femoral metaphyseal osteomyelitis, which may result in true septic arthritis, will always manifest an early 'sympathetic' effusion and if so whether this would yield organisms on aspiration.

Fink et al state that 'despite hospital admission' delayed diagnosis of septic arthritis occurs since aspiration is delayed until patients have deteriorated. This proved not to be the case in our series, which included 21 patients with sepsis whereas they encountered only one. All our cases of septic arthritis declared themselves clinically and the diagnostic difficulties only occurred with pelvic osteomyelitis, which was suspected and promptly demonstrated by scintigraphy.

There is widespread agreement in the literature that laboratory and clinical signs in isolation are poor at distinguishing irritable hip from septic conditions. However, the conclusion of our review was that in combination certain of these factors became far more sensitive and yet retained sufficient specificity to avoid unnecessary admissions. Similarly, Del Beccaro et al, quoted by Fink et al, stated that a sedimentation rate of $>20 \mathrm{~mm} /$ hour and a fever of $37.5^{\circ} \mathrm{C}$ identified all but one case of septic arthritis out of 38 cases and should lead to consideration of a diagnostic aspiration. ${ }^{3}$ Although aspiration may be preceded by a confirmatory ultrasound, as long as this does not lead to undue delay, the technique is not $100 \%$ accurate as shown by McGoldrick et al ${ }^{1}$ and Egund et al. ${ }^{4}$

Whether or not there is a prolonged therapeutic effect from aspiration of painful effusions is unproved. The largest series of irritable hip patients treated by aspiration are those of Kallio et $a l^{5}$ and Erken and Katz, ${ }^{6}$ who aspirated and measured intracapsular pressures in 97 and 85 patients respectively. Both of these studies related the volume of the aspirate to the severity of symptoms and the former to the ultrasound imaged distension of the capsule. The intracapsular pressure was thought to be the key factor and this depended upon volume and hip position. They, along with Wingstrand ${ }^{7}$ and Fink et al have all shown that aspiration is beneficial in reducing immediate pain, but no prospective study has comparatively addressed the influence of this, or any other treatment including hospital 